\title{
CHOLESTEATOME CONGENITAL DE L'OREILLE MOYENNE A PROPOS DE DEUX CAS
}

\author{
J. KOUBAA, F. BEN MAHMOUD, S. KADI, I. GASSAB, K. HARRATHI, A. BERKAOUI, M. GOLLI*, \\ F. EL KADHI, A. GASSAB. \\ SERVICE ORL ET CHIRURGIE CERVICOFACIALE \\ *SERVICE D'IMAGERIE MÉDICALE \\ HÔPITAL FATTOUMA BOURGUIBA. MONASTIR. TUNISIE
}

\begin{abstract}
But : Le cholestéatome congénital de l'oreille moyenne est une entité rare. L'objectif de ce travail est de discuter les particularités cliniques et thérapeutiques de cette pathologie à travers deux observations de malades présentant un cholestéatome congénital opérés et suivis au service d'ORL et de chirurgie cervico-faciale Fattouma Bourguiba Monastir, entre 2002 et 2008.

Résultats : II s'agissait de deux garçons, âgés de 5 et 7 ans. La symptomatologie était dominée par l'apparition d'une hypoacousie et l'examen otologique a révélé un tympan complet dans les deux cas. La TDM des rochers a permis d'évoquer le diagnostic du cholestéatome congénital et d'apprécier les extensions locales des lésions. Les deux malades ont eu une tympanoplastie en technique fermée.

Conclusion : L'évolution du cholestéatome congénital est insidieuse et le diagnostic est souvent tardif. Le traitement est chirurgical, dominé par la tympanoplastie en technique fermée.
\end{abstract}

Mots clès : Chlolestéatome congénital, hypoacousie, tympan complet, tympanoplastie en technique fermée, technique ouverte.

\section{SUMMARY}

AIM : Congenital cholesteatoma of the middle ear is a rare entity. The objective of this study is to discuss clinical and therapeutic particularities of this pathology through 2 cases operated in the ENT department of Fattouma Bourguiba Hospital of Monastir, between 2002 and 2008.

Résultats : They were two males aged 5 and 7 years. Main complains was hearing loss with a complete tympanic membrane in clinical exam. CT scan had confirmed the diagnosis of congenital cholesteatoma and had evaluated the cholesteatoma local extension. Canal wall-up tympanoplasty was performed for both patients.

Conclusion : Diagnostic of congenital cholesteatoma is late and difficult due the long latency period. The surgical treatment is based in the canal wall-up tympanoplasty.

Key words : Congenital cholesteatoma, hearing loss, complete tympanic membrane, canal wall-up tympanoplasty, canal wall-down tympanoplasty

\section{INTRODUCTION}

Le cholestéatome congénital ou primitif de l'oreille moyenne est une entité clinique rare. II représente selon les séries entre 5 à $15 \%$ des cholestéatomes de l'enfant (1-3).

Son existence, bien que décrite pour la première fois en 1830 par Korner cité par Potsic et al. (1) a longtemps été contestée mais elle est actuellement admise par la majorité des auteurs $(2,3)$.

Après la publication des critères diagnostiques des cholestéatomes congénitaux par Derlacki, cette pathologie est devenue mieux connue et identifiée par les oto-rhinolaryngologistes et les pédiatres (2).

\section{OBSERVATION No 1}

Il s'agissait de l'enfant S.M âgé de 7 ans, sans antécédents pathologiques notables présentant une hypoacou- sie et des acouphènes gauches d'apparition progressive et évoluant depuis 2 ans sans notion de vertige ni d'otorrhée associés.

L'examen otoscopique de l'oreille gauche complété par un examen sous microscope, a révélé un tympan complet, refoulé par une formation blanchâtre rétro-tympanique.

L'examen de l'oreille controlatérale, ainsi que l'examen neuro-vestibulaire étaient normaux. L'audiométrie tonale a objectivé une surdité de transmission de $35 \mathrm{~dB}$ à gauche et une audition normale à droite.

La TDM des rochers a montré un comblement tissulaire de l'oreille moyenne gauche refoulant les osselets en haut avec éburnation des cellules mastoïdiennes du même côté évoquant un cholestéatome. (Fig. 1) 


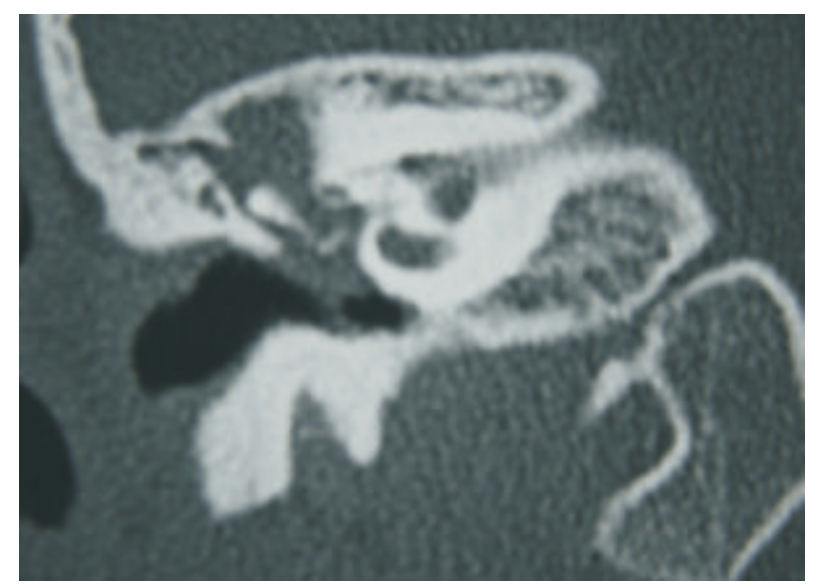

Fig 1 : TDM des rochers: Comblement tissulaire de l'oreille moyenne.

Le malade a été opéré par voie rétro-auriculaire. En peropératoire, on a remarqué la présence d'un cholestéatome comblant la caisse, l'antre ainsi que l'attique antérieur et postérieur. La chaîne ossiculaire était complète.

Le malade a eu une tympanoplastie en technique fermée avec tympanotomie antérieure et postérieure et exérèse du cholestéatome. Cette exérèse était satisfaisante sauf au niveau des branches de l'étrier.

Douze mois plus tard, le malade a bénéficié d'un second look : il existait une récidive du cholestéatome qui comblait toutes les cavités.

Une exérèse complète du cholestéatome et une transformation de la technique fermée en technique ouverte ont été réalisées, suivies d'un aménagement d'une minicaisse et d'une méatoplastie.

Les suites opératoires étaient simples. La cavité est actuellement stable sans récidive avec un recul de 4 ans.

\section{OBSERVATION No 2}

II s'agissait d'un enfant âgé de 5 ans, aux antécédents d'une méningite à pneumocoque traitée il y a un an. Ce patient nous a été adressé pour exploration d'une hypoacousie bilatérale, signalée par les parents, sans vertige ni signes neurologiques associés.

L'examen otoscopique a révélé un tympan complet avec présence d'un bombement important au niveau des cadrans antérieur et postérieur du côté gauche, évoquant un cholestéatome congénital. Du coté controlatéral, on a noté un aspect d'otite séro-muqueuse. L'examen neurovestibulaire était sans anomalies.

Le bilan audiométrique a montré la présence d'une surdité de transmission bilatérale avec un seuil de $50 \mathrm{~dB}$ à gauche et $35 \mathrm{~dB}$ à droite.

La TDM des rochers a objectivé un comblement de la caisse du tympan des deux côtés et des cellules mastoïdiennes du côté gauche. (Fig 2)

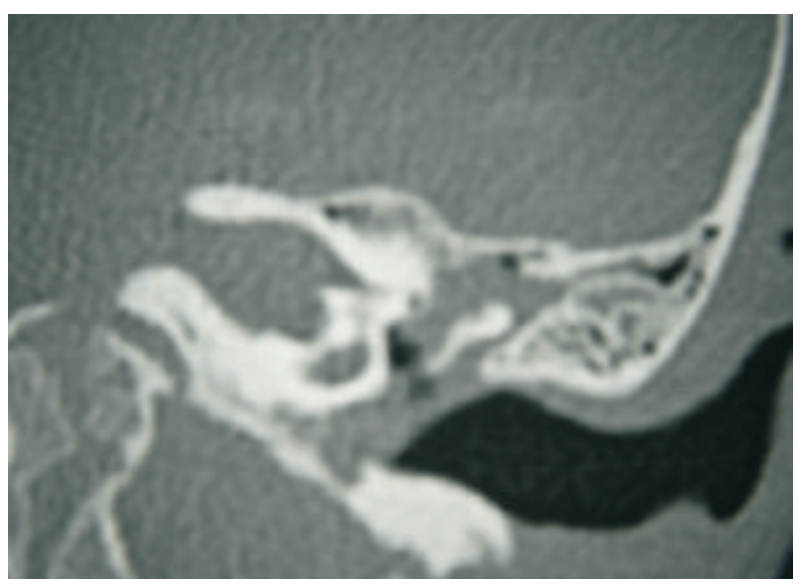

Fig 2 : TDM des rochers: Comblement de la caisse du tympan du côté gauche

L'examen sous microscope peropératoire a montré un bombement important du tympan à gauche et un aspect d'otite séromuqueuse à droite.

La paracentèse a révélé la présence de cholestéatome en rétro-tympanique du côté gauche et une issue de glue du côté droit.

Le malade a été opéré par voie rétro-auriculaire du côté gauche. Le cholestéatome occupait toute la caisse, l'attique antérieur et postérieur ainsi que l'antre et englobait la chaîne ossiculaire qui était complète.

Après déarticulation incudostapédienne et ablation du marteau et de l'enclume, nous avons réalisé une tympanotomie postérieure permettant une exérèse satisfaisante du cholestéatome.

La reconstruction du tympan a été faite par un totocartilage reposant sur le bouton de l'étrier, réalisant une myringostapédopexie cartilagineuse.

En fin d'intervention, la mise d'un aérateur transtympanique du côté controlatéral a été faite. Les suites opératoires étaient simples.

Une TDM des rochers pratiquée après un délai de 12 mois, n'a pas révélé de récidive cholestéatomateuse (Fig 3)

Le patient a été admis 3 mois après pour oto-mastoidïte gauche qui a bien évolué sous traitement antibiotique à base de Claforan* ${ }^{\star}(200 \mathrm{mg} / \mathrm{kg} / \mathrm{j})$, Fosfomycine* (200 $\mathrm{mg} / \mathrm{kg} / \mathrm{j})$, Flagyl ${ }^{*}$ (30 mg/kg/j).

Un second look a été donc indiqué. En per-opératoire, on n'a pas retrouvé de récidive cholestéatomateuse. 


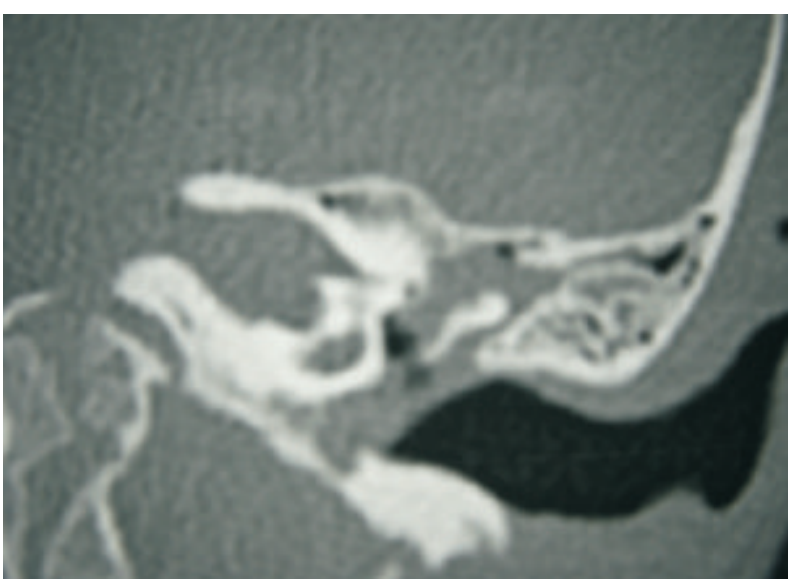

Fig 3 : TDM des rochers post-opératoire : Absence de signes évidents de récidive cholestéatomateuse.

\section{DISCUSSION}

Le cholestéatome congénital de l'oreille moyenne est une entité clinique rare. II représente selon les séries entre 5 à $15 \%$ des cholestéatomes de l'enfant (1-3).

Cette fréquence est en nette augmentation actuellement du fait d'un diagnostic plus précoce (4).

L'étiopathogénie la plus admise actuellement est celle de Leslie Michaels qui suppose la persistance d'une formation épidermique embryonnaire au niveau de la zone antéro-supérieure de la paroi externe de la caisse du tympan qui doit normalement involuer à la 33ème semaine de gestation.

Cette théorie expliquerait la localisation typique, au niveau du promontoire, des cholestéatomes congénitaux au début de leur évolution (5-6).

D'autres théories ont été proposées notamment (7) :

- Une migration des cellules épidermiques embryonnaires à travers le bourrelet annulaire de Gerlach.

- Un reflux du liquide amniotique contenant des cellules épithéliales, par la trompe auditive, lors de l'accouchement.

L'âge moyen des enfants au moment du diagnostic est compris entre 4 et 8 ans $(8,9)$. Effectivement, nos observations ont concerné deux patients âgés de 5 et 7 ans. Néanmoins, le cholestéatome congénital peut être diagnostiqué très tôt, dès les premiers mois ou semaines de la vie (à partir de la 6ème semaine pour Schwartz et al.) ou à un âge adulte a la cinquantaine d'après une série présenté par Kojima et al. $(10,11)$.

Une prédominance masculine est notée dans la majorité des séries publiées avec un sexe ratio de $3 / 1(1,3)$. La cause de cette prédominance masculine demeure inconnue.

La présentation clinique du cholestéatome congénital est très différente des cholestéatomes acquis.

Les cholestéatomes congénitaux de l'oreille moyenne sont diagnostiqués souvent lors du bilan d'une hypoacou- sie unilatérale et ceci dans 60 à $65 \%$ des cas selon les séries $(7,11)$. L'antécédent d'une otite séro-muqueuse résistante au traitement médical, des épisodes d'otorrhée ou d'otalgie peut également être noté au cours des cholestéatomes congénitaux (12).

Le diagnostic est parfois posé au moment d'une paracentèse ou de la pose d'un aérateur transtympanique, lorsque l'aspiration ramène des squames épidermiques $(8,12,13)$.

La présence d'une complication, notamment une paralysie faciale périphérique, est rarement révélatrice du cholestéatome congénital. Cette circonstance est observée dans moins de $1 \%$ des cas selon William et al. (14).

Des formes asymptomatiques révélées lors d'un examen systématique sont également décrites $(11,14)$.

A l'examen sous microscope, le tympan est intègre avec présence d'une masse blanchâtre rétrotympanique localisée le plus souvent au niveau du quadrant antérosupérieur (7). Lorsque toute la caisse est atteinte, la présentation otoscopique est celle d'un «tympan blanc ». C'est la forme la plus souvent décrite par Darrouzet et al. (3). Le diagnostic du cholestéatome congénital est difficile à établir devant la rareté de cette pathologie et l'absence d'une symptomatologie spécifique orientant vers ce diagnostic. Pour ceci, des critères cliniques ont été posés par Derlacki et Clemis depuis1965, permettant de définir cette entité (2) :

- Le développement derrière un tympan normal.

- L'absence d'antécédents d'otite, d'otorrhée ou de perforation tympanique.

- L'absence de tout acte invasif sur le tympan (paracentése, aérateur transtympanique, traumatisme).

Mais devant l'incidence importante des otites chez les enfants de cet âge, Levenson(1988) et Parisier(1989) ont retiré l'absence d'antécédents otitiques parmi les critères diagnostiques (15).

Des malformations congénitales associées au cholestéatome congénital ont été rapportées dans la littérature, notamment les malformations de la chaîne ossiculaire (absence de l'enclume, présence d'un bloc marteauenclume, atrophie ou absence de l'étrier) mais également les malformations cervico-faciales (fente vélopalatine, kystes et fistules congénitaux du cou) et les malformations abdomino-pelviennes (malformations urogénitales, sténoses du pylore, luxations de la hanche, dysgerminome de la glande pinéale...) (8).

Selon Levenson et Parisier, la présence d'une malformation ossiculaire associée à un cholestéatome congénital est retrouvée dans environ $15 \%$ des cas (15).

L'imagerie notamment la TDM des rochers permet de poser le diagnostic en montrant une opacité sphérique rétrotympanique, d'établir les extensions locorégionales du cholestéatome et de préciser les atteintes ossiculaires associées. Une étude minutieuse du côté controlatérale doit être systématiquement réalisée à la recherche de 
malformations associées mais surtout de cholestéatome bilatéral (12).

Cette éventualité est exceptionnelle puisque moins de 15 cas de cholestéatomes bilatéraux ont été décrits dans la littérature. La méconnaissance d'une forme bilatérale, bien qu'exceptionnelle, engage davantage le pronostic fonctionnel que dans les atteintes unilatérales $(16,17)$.

Deux classifications des cholestéatomes congénitaux sont actuellement admises. La première en distingue 2 types $(3,18)$ :

- Les cholestéatomes encapsulés, localisés au niveau du quadrant antéro-supérieur avec un retentissement fonctionnel peu important, de découverte souvent fortuite.

- Les cholestéatomes diffus, mésotympaniques, se développant vers l'épitympanum, les cavités postérieures et conduisant très souvent à une lyse ossiculaire. Ils sont souvent révélés par une surdité de transmission sévère ou par des complications, c'est le cas de nos malades.

Une deuxième classification a été proposée lors du seizième congrès annuel de la société américaine d'ORL pédiatrique en 2001. Elle prend en considération le ou les quadrants envahis ainsi que l'atteinte ossiculaire et permet de distinguer 4 stades évolutifs, ayant chacun un pronostic et un traitement différent $(13,19)$ :

- Stade I : atteinte d'un seul quadrant ;

- Stade II : atteinte de plusieurs quadrants sans atteinte ossiculaire ;

- Stade III : atteinte ossiculaire (qu'elle soit secondaire à la lyse par le cholestéatome ou à une chirurgie préalable); - Stade IV : extension à la mastoïde.

Selon ces mêmes auteurs, le taux de cholestéatomes résiduels est plus important dans le stade IV.

Le cholestéatome congénital est caractérisé par un potentiel évolutif et destructif important justifiant une prise en charge chirurgicale adaptée et précoce.

Les deux objectifs du traitement sont l'éradication complète et définitive du cholestéatome et la restauration de la fonction auditive chez le jeune patient en plein apprentissage.

La technique fermée est privilégiée selon la majorité des auteurs $(8,18)$.

Selon une série présentée par Benhammou comportant 33 malades présentant des cholestéatomes congénitaux, $88 \%$ d'entres eux ont eu des tympanoplasties en technique fermée (7).

Selon une série portant sur 119 cas de cholestéatomes congénitaux, $96 \%$ des malades ont eu des tympanoplasties en technique fermée (13).

Ce choix est justifié par les caractéristiques particulières du cholestéatome congénital de l'enfant. La pneumatisation mastoïdienne est souvent normale; la muqueuse de la cavité tympanique est généralement saine avec une fonction tubaire normale.

Lorsque le contrôle du cholestéatome est satisfaisant et en présence d'une cavité tympanique non inflammatoire, l'ossiculoplastie pourrait être réalisée dans le même temps opératoire.

L'absence ou la présence de lyse ossiculaire sont très variables selon les séries. Conraux (20) retrouve $80 \%$ des chaînes ossiculaires lysées alors que Levenson ne retrouve que $20 \%$ (21).

Les résultats fonctionnels sont différents selon les séries publiées compte tenu de la variabilité des populations, des stades lésionnels, du statut ossiculaire et du choix de la technique chirurgicale.

Selon les auteurs, le gain auditif est fonction de l'atteinte ossiculaire, de la technique de l'ossiculoplastie réalisée et de l'expérience du chirurgien $(11,13)$.

Dans la plupart des séries, la présence ou non de l'étrier constitue un facteur prédictif du résultat fonctionnel, l'audition étant meilleure en cas d'étrier présent (Rinne résiduel entre 15 et $20 \mathrm{~dB}$, alors qu'il est de 30 à $40 \mathrm{~dB}$ lorsque l'étrier est absent) $(11,18,22)$.

Selon Shirazi, il n'y a pas de différence significative du gain auditif selon la technique chirurgicale adoptée (tympanoplastie en technique fermée ou en technique ouverte)(23).

Un deuxième temps opératoire est systématique pour la plupart des auteurs (révision plus ou moins temps fonctionnel). Ce temps est réalisé dans 65 à $85 \%$ des cas selon les séries $(7,13)$.

Selon Nelson, le second look n'est pas nécessaire en cas de cholestéatome congénital stade 1 puisque le pourcentage de cholestéatome résiduel à ce stade est très faible (13).

Un deuxième temps opératoire a été réalisé chez nos deux malades, après un délai de 12 et 20 mois.

L'imagerie (TDM et IRM) gagne de plus en plus d'intérêt dans la détection du cholestéatome résiduel au cours de la surveillance post-opératoire. La TDM des rochers peut affirmer l'absence de cholestéatome lorsqu'elle est normale, mais elle ne peut ni prédire ni exclure un cholestéatome résiduel devant la présence d'une opacité. De même I'IRM permet de suspecter des résiduels de plus de $2 \mathrm{~mm}$, mais les aspects décrits ne sont pas spécifiques $(12,24)$.

La fréquence des cholestéatomes résiduels après chirurgie est très variable dans la littérature.

Le taux de cholestéatome résiduel est de $25 \%$ des cas selon la série de Benhammou (7). Dans les séries américaines, ce taux est de 10 à $15 \%$ des cas $(13,25)$.

Selon une série japonaise portant sur 63 patients présentant des cholestéatomes congénitaux, seulement 3.9\% des malades qui ont eu des tympanoplasties en technique fermée ont présenté des cholestéatomes résiduels (11). Trois facteurs prédictifs seraient étroitement et individuellement associés à la survenue de cholestéatome résiduels: l'envahissement de l'atrium postérieur, l'existence d'une lyse ossiculaire et la présomption d'une ablation incomplète du cholestéatome (26). 
Selon William, la présence d'un cholestéatome résiduel est dépendant du stade initial des lésions : il est de 14\% pour le stade I, $33 \%$ pour le stade II, $41 \%$ pour le stade III et de $67 \%$ pour le stade IV d'après une étude portant sur 167 cas de cholestéatomes congénitaux (14).

\section{CONCLUSION}

Le cholestéatome congénital est une entité clinique rare, différente du cholestéatome acquis. Ce type de cholestéatome semble évoluer de façon plus rapide et être plus agressif.

Cette entité pose un problème diagnostique puisqu'elle se révèle par une symptomatologie clinique peu spécifique. Le traitement de choix des cholestéatomes congénitaux est la tympanoplastie en technique fermée avec tympanotomie postérieure et ossiculoplastie.

Cette attitude permet de répondre à deux objectifs : l'exérèse définitive du cholestéatome et la conservation d'une audition utile garant le bon développement du langage et la bonne intégration sociale de l'enfant.

\section{REFERENCES}

1. Potsic WP, Korman SB, Samadi DS, Wetmore RF. Congenital cholesteatoma: 20 years' experience at The Children's Hospital of Philadelphia. Otolaryngol Head Neck Surg 2002; 126: 409-14.

2. Derlacki EL, Clemis JD. Congenital cholesteatoma of the middle ear and mastoid. Ann Otol Rhinol Laryngol 1965; 74:706-27.

3. Darrouzet V, Duclos JY, Portmann D, Bebear JP. Congenital middle ear cholesteatomas in children: our experience in 34 cases. Otolaryngol Head Neck Surg 2002; 126:34-40.

4. Lerosey Y, Andrieu-Guitrancourt J, Marie JP, Dehesdin D. Le cholestéatome de l'oreille moyenne chez l'enfant. Critères de décision chirurgicale. A propos de 57 cas. Ann Otolaryngol Chir Cervicofac 1998; 115:215-21.

5. Bernal-Sprekelsen M, Sudhoff H, Hildmann H. Evidence against neonatal aspiration of keratinizing epithelium as a cause of congenital cholesteatoma. Laryngoscope 2003; 113:449-51.

6. Kayhan FT, Mutlu C, Schachern PA, Le CT, Paparella MM. Significance of epidermoid formations in the middle ear in fetuses and children. Arch Otolaryngol Head Neck Surg 1997; 123: 1293-7.

7. A. BenHammou, Nguyen D.Q., K. El Makhloufi, R. Charachon, E. Reyt, S. Schmerber Résultats à long-terme des cholestéatomes congénitaux de l'oreille moyenne de l'enfant Ann Otolaryngol Chir Cervicofac, 2005 ; 122, 3, 113-119 8. Duclos JY, Darrouzet V, Portmann D, Portmann M, Bebear JP. Cholestéatomes congénitaux de l'oreille chez l'enfant. Analyse clinique, évolutive et thérapeutique d'une série de 34 cas. Ann Otolaryngol Chir Cervicofac 1999; 116:218-27.

9. Besbes G., Kharrat S., Trabelsi S., Beltaief N., Sahtout S., Hachicha S.. Le cholestéatome congénital de l'oreille moyenne : A propos de deux cas. J .TUN ORL 2006; 17 :35-37.

10. Schwartz RH, Grundfast KM, Mcaveney WJ, Merida MA, Feldman B. Congenital middle ear cholesteatoma. Am J Dis Child 1983; 137:501-2.

11. Kojima H, Tanaka Y, Shiwa M. Congenital cholesteatoma: clinical features and surgical results. American Journal of Otolaryngology Head and Neck Medicine Surgery 2006; 27: 299- 305.

12. El Bitar M. A., Choi SS. Congenital middle ear cholesteatoma: need for early recognition role of computed tomography scan. International Journal of Pediatric Otorhinolaryngology 2003; 67: 231-5.

13. Nelson M, Roger G, Koltai PJ, et al. Congenital cholesteatoma: classification, management, and outcome. Arch Otolaryngol Head Neck Surg 2002; 128:810-4.
14. William P, Bruce S, Daniel S., RALPH F. Congenital cholesteatoma: 20 years' experience at the children's Hospital of Philadelphia. Otolaryngol Head Neck Surg 2002; 126: 409-14.

15. Parisier SC, Levenson MJ, Edelstein DR, et al. Management of congenital pediatric cholesteatomas. Am J Otol 1989; 10:121-3.

16. Litman RS, Smouha E, Sher WH, Shangold LM. Two cases of bilateral congenital cholesteatoma -usual and unusual presentations. Int J Pediatr Otorhinolaryngol 1996; 36:241-52.

17. El-Bitar MA, Choi SS. Bilateral occurrence of congenital middle ear cholesteatoma. Otolaryngol Head Neck Surg 2002; 127: 480-2.

18. Charachon R, Gratacap B, Fillatre B. Le cholestéatome congénital de l'oreille moyenne de l'enfant. Rev Laryngol Otol Rhinol 1992; 113:7-10.

19. Potsic WP, Samadi DS, Marsh RR, Wetmore RF. A staging system for congenital cholesteatoma. Arch Otolaryngol Head Neck Surg 2002; 128:100912.

20. El JErrari A, Stierle JL, Debry C, Veillon F, Gentine A, Conraux C. Le cholestéatome congénital et les malformations ossiculaires associées. Ann Otolaryngol Chir Cervicofac $1995 ; 112$ : 258-61.

21. Levenson MJ, Parisier SC, Chute P, Wenig S, Juarbe C. A review of twenty congenital cholesteatomas of the middle ear in children. Otolaryngol Head Neck Surg $1986 ; 94: 560-7$

22. Soderberg KC, Dornhoffer JL. Congenital cholesteatoma of the middle ear: occurrence of an "open" lesion. Am J Otol 1998; 19:37-41.

23. Shirazi MA, Muzaffar K, Leonetti JP. Surgical Treatement of PediatricCholesteatoma. Laryngoscope 2006; 116: 1603-7.

24. Denoyelle F, Silberman B, Garabedian EN, intérêt de l'imagerie par résonance magnétique couplée à la tomodensitométrie dans le dépistage des cholestéatomes résiduels après chirurgie primaire. Ann otolaryngol chir cervicofac 1994; 111: 85-8.

25. Liu JH, Rutter MJ, Choo DI, Willging JP. Congenital cholesteatoma of the middle ear. Clin Pediatr (Phila) 2000; 39:549-51.

26. Roger G, Schlegel N, Chauvin P, Denoyelle F, Garabedian EN. Facteurs prédictifs de la survenue de cholestéatomes résiduels chez l'enfant. Ann Otolaryngol Chir Cervicofac 1995; 112:262-74. 